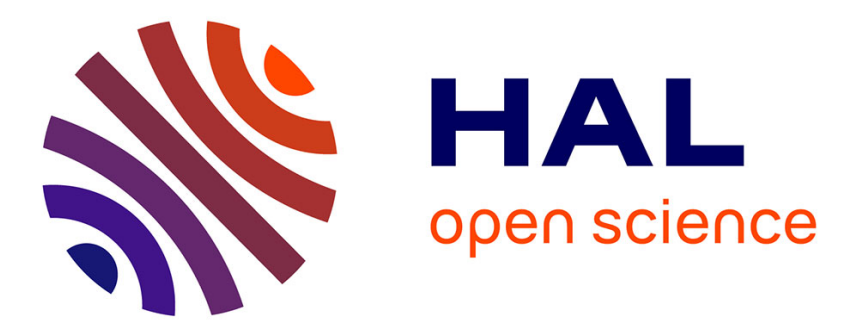

\title{
The VIRGO Challenge: Detecting Strain Amplitudes Smaller Than 10-21
}

G. Cagnoli, L. Gammaitoni, J. Kovalik, F. Marchesoni, M. Punturo

\section{To cite this version:}

G. Cagnoli, L. Gammaitoni, J. Kovalik, F. Marchesoni, M. Punturo. The VIRGO Challenge: Detecting Strain Amplitudes Smaller Than 10-21. Journal de Physique IV Proceedings, 1996, 06 (C8), pp.C8-833-C8-836. 10.1051/jp4:19968179 . jpa-00254615

\section{HAL Id: jpa-00254615 https://hal.science/jpa-00254615}

Submitted on 1 Jan 1996

HAL is a multi-disciplinary open access archive for the deposit and dissemination of scientific research documents, whether they are published or not. The documents may come from teaching and research institutions in France or abroad, or from public or private research centers.
L'archive ouverte pluridisciplinaire HAL, est destinée au dépôt et à la diffusion de documents scientifiques de niveau recherche, publiés ou non, émanant des établissements d'enseignement et de recherche français ou étrangers, des laboratoires publics ou privés. 


\title{
The VIRGO Challenge: Detecting Strain Amplitudes Smaller Than $10^{-21}$
}

\author{
G. Cagnoli, L. Gammaitoni, J. Kovalik, F. Marchesoni and M. Punturo
}

Istituto Nazionale di Fisica Nucleare, VIRGO-Project, Universita' di Perugia, 06100 Perugia, Italy

\begin{abstract}
The French-Italian collaboration VIRGO is presently building an interferometric gravitational wave detector aimed at measuring spectral strains in vacuo as small as $10^{-23} / \sqrt{\mathrm{Hz}}$ in the bandwidth $5-10^{4} \mathrm{~Hz}$. Elimination of thermal noise effects poses new challenges to internal friction experts. VIRGO requirements and strategies are outlined here with the purpose of stimulating discussion, advice and, possibly, collaboration.
\end{abstract}

\section{THE VIRGO ANTENNA}

Gravitational waves (GW) are deformations of the space-time geometry [1]: the effect of a GW on two free floating masses of given space-time coordinates is a time-dependent modulation of their distance $L$ with amplitude $\Delta L$. Being quadrupolar fields (with two orthogonal polarizations), GW are emitted mostly by such astrophysical objects as coalescing binaries, asymmetric supernovae explosions, rotating neutron stars with quadrupole moment, etc. The strength of a GW field is defined by the local strain amplitude $h=2 \Delta L / L$ (dimensionless) or by its spectral component $\tilde{h}(\nu)$ (in units of $1 / \sqrt{\mathrm{Hz}}$ ). For the favorable case of two black-holes of one solar mass each spiralling through a distance of $3 \mathrm{Km}$ at $10 \mathrm{Mpc}$ from us, we expect a strain amplitude at the earth surface as small as $10^{-21}$ (i.e. $\tilde{h}=10^{-23} / \sqrt{\mathrm{Hz}}$ ): extremely sensitive antennas are required to monitor GW sources. The first experiments for the detection of GW made use of aluminum bars [1], whose high-frequency internal modes ought to be excited by GW pulses (with $\tilde{h} \sim 10^{-17} / \sqrt{\mathrm{Hz}}$ ). Alternative, though more challenging, detection schemes may be construed by having recourse to a Michelson interferometer. An incident GW, if properly polarized, stretches one interferometer arm with respect to the other; the resulting arm length difference $\Delta L$ can be detected at the interferometer (dark fringe) output. Since no internal resonances are involved, a large detection bandwidth is expected $\left(5-10^{4} \mathrm{~Hz}\right.$ for VIRGO). The antenna sensitivity can be enhanced by taking longer arms, because $\Delta L$ is proportional to the arm length $L$. However, if $L$ exceeds one fourth of the GW wavelength, $\Delta L$ reverses sign before the light interference path is completed, and the sensitivity decreases. The optimal condition may be achieved by making the light perform several roundtrips in each arm before coming back to the beam splitter (optical delay line). VIRGO alternative design consists in inserting a Fabry-Perot cavity in each interferometer arm. In both cases, the effective light path is increased, whereas the physical arm length $L$ can be scaled down to $3 \mathrm{Km}$. For smaller $L$ values, thermal fluctuations in the optical components supersede the GW 
signal $\Delta L(t)$.

The VIRGO antenna [2] is presently under construction in Italy with the participation of laboratories from the French CNRS (Annecy, Lyon, Orsay, Paris) and the Italian INFN (Frascati, Napoli, Perugia, Pisa, Roma). The design of a laser interferometric GW detector is largely determined by the noise sources one wants to minimize. The expected sensitivity curve of the VIRGO antenna is shown in Fig. 1, where the effect of each noise source is plotted separately.

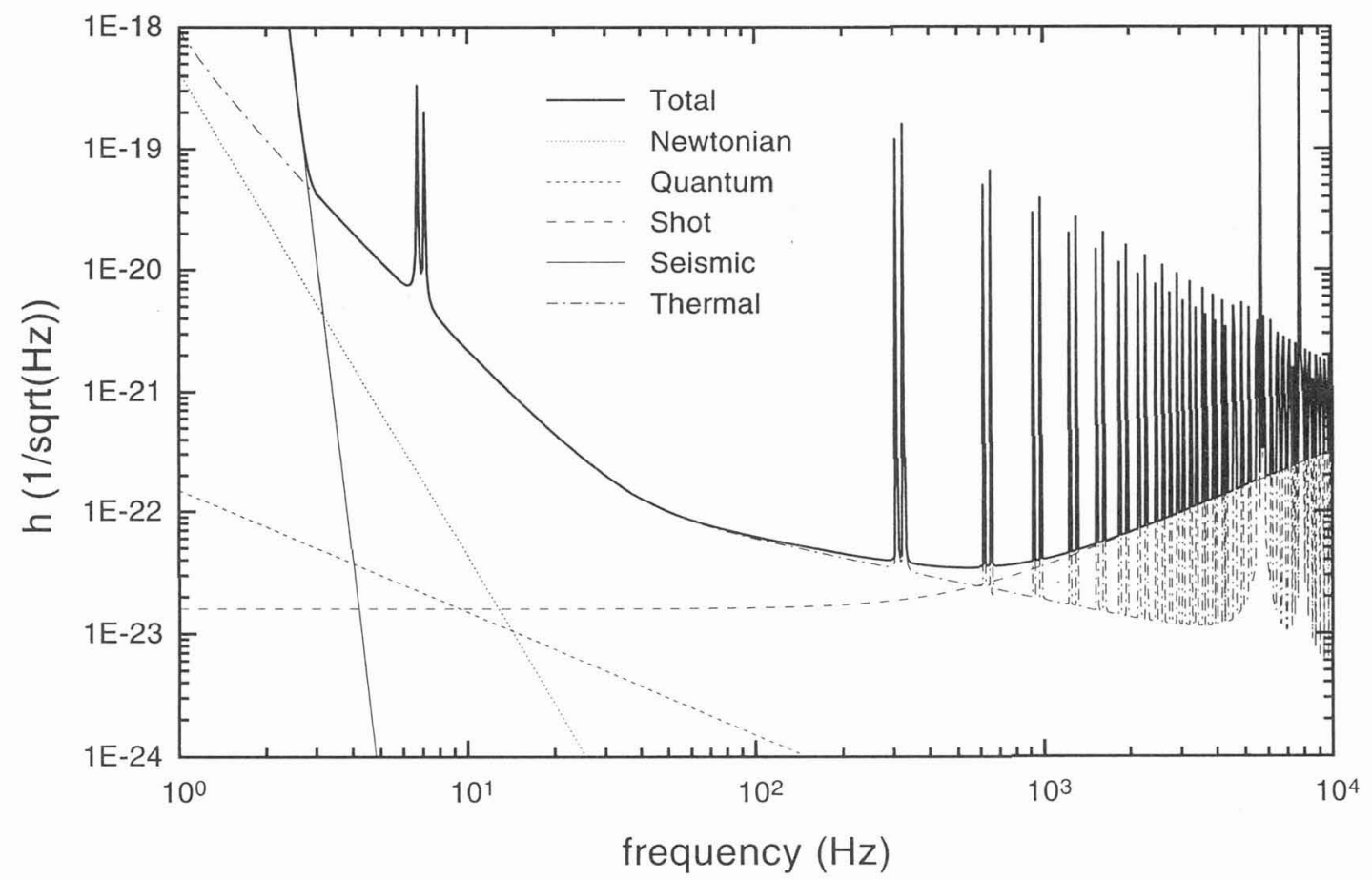

Figure 1: Expected VIRGO sensitivity curve.

(a) Seismic noise. Since the mirrors are not free falling masses, undesired ground vibrations due to seismic and human activities must be eliminated. All sensitive optical components are suspended to seven-stage pendulums (superattenuators), where each stage acts as a mechanical linear seismic filter. Hence, all VIRGO superattenuators have an above resonance seismic impedance proportional to $\nu^{-14}$; seismic noise is, thus, effectively filtered out for $\nu>3 \mathrm{~Hz}$.

(b) Thermal noise. The amount $k T$ of thermal energy stored in each mode of a classical system at equilibrium, determines the amplitude of its fluctuations. The most disturbing consequence is a wide-band off-resonance spectral noise background, which limits the VIRGO sensitivity in the $3-200 \mathrm{~Hz}$ range. In particular, mirror internal modes, which cause its surface to warp, mimic time modulations of $L$. Thermal noise also causes the suspended mirror to oscillate, and excites violin modes in the suspension wires. Thermal noise can be minimized by selecting low internal friction (IF) materials and an appropriate mirror design (a $25 \mathrm{Kg}$ cylinder suspended by two wire loops).

(c) Shot noise. Phase and amplitude of the light field are conjugate variables and cannot be 
determined simultaneously with arbitrary precision; therefore, the power of a light beam impinging on a photodiode is expected to fluctuate and the relevant photocurrent exhibits electronic shot noise. The spectrum of the equivalent arm-length fluctuations is $(\lambda / 4 \pi) \tilde{\phi}$, where $\tilde{\phi}(\nu)$ is the light phase spectrum $\sqrt{h \nu / P}(P, \nu$ and $\lambda$ are the laser power, frequency and wavelength). Obviously, the shot noise can be reduced by increasing the laser power. Note that for the realistic value $P=10 \mathrm{~W}$, radiation pressure fluctuations are negligible.

(d) Residual gas. Refractive index fluctuations due to residual gases in the interferometer cavities might be mistaken for a GW signal. The tolerable residual pressure is estimated of the order of $10^{-9} \mathrm{mbar}$ for hydrogen and $10^{-10} \mathrm{mbar}$ for other gases. The VIRGO steel tubes will be thermally treated at $400 \mathrm{C}$ by the manufacturer to reduce the hydrogen outgassing rate, and then backed at $150 \mathrm{C}$ after assembling, mostly to remove water vapor. The VIRGO interferometer will be the biggest high-vacuum cavity ever built.

(e) Scattered light noise. Light scattered at the mirror surfaces may hit the wall of the grounded vacuum tube, where it would pick up seismic noise; a certain fraction of noisy light is eventually reinjected into the main beam through subsequent scattering processes. It is therefore of critical importance to develop extremely high quality mirrors with low scattering losses $(0.5 \mathrm{ppm})$.

(f) Laser noise. In the ideal case of perfectly symmetric arms, a Michelson interferometer is insensitive to laser noise, which would cancel out at the output. Spurious asymmetries, however, determine a certain degree of sensitivity to laser imperfections. Hence, the laser beam injected into the interferometer must be of exceptionally good quality: an infrared $(1.06 \mu \mathrm{m})$ high-power $(10 \mathrm{~W})$ laser cavity locked with frequency stability of $10^{-2} \mathrm{~Hz} / \sqrt{\mathrm{Hz}}$ at $500 \mathrm{~Hz}$ and equipped with a mode cleaner cavity to eliminate beam jitter and guarantee good geometry.

\section{THE ROLE OF INTERNAL FRICTION (IF)}

Under the denomination of thermal noise, item (b), one ought to include most dissipation mechanisms listed in the programme of this Conference. VIRGO extreme requirements on the mechanical components of the antenna might well catch the attention of IF experts. We mention, here, a few examples:

(1) Low frequency losses. Sketchy evidence [3-4] and preliminary tests support the idea that the intrinsic loss angle $\phi(\omega)$ in most metallic samples including commercial steels, Cu-Be alloys, etc., is frequency independent below $10^{3} \mathrm{~Hz}$. Bulk $1 / f$ mechanical noise can be traced back to the stick-and-slip nature of the relaxation processes involving pinned dislocations [4-6]. Curve (b) in Fig. 1 was drawn on assuming $\phi=310^{-4}$. Note that a viscous loss $\phi(\omega) \sim \omega$ would improve the antenna sensitivity at low frequencies (a VIRGO priority, indeed!), whence our interest in a systematic determination of the function $\phi(\omega)$ in the frequency range $1-10^{3} \mathrm{~Hz}$.

As a further complication of our suspension design, we remember that each loop wire is loaded up to $65 \%$ of its breaking point (with stress $\sigma_{b}$ ). The question rises, then, as how a strong static tensile stress $\sigma_{l}$ affects the loss angle driven by a weak periodic bias $\sigma_{0} \cos \omega t$. Tests performed on annealed commercial steel samples in both torsional and inverted pendulums with $\sigma_{0} \ll \sigma_{l} \leq 0.5 \sigma_{b}$ show a very weak load dependence of $\phi(\omega)$. The same conclusion was reached for $\mathrm{Cu}-\mathrm{Be}$ and other alloys [4]. A satisfactory explanation of these results proved elusive, so far.

(2) Mechanical shot noise. Although $\phi(\omega)$ seems to be independent of $\sigma_{l}$, we do pay a price for choosing a very thin cross-section for our suspension wires: the stationary creep mechanism at room temperature $T=300 \mathrm{~K}$ (stability $\pm 0.5 \mathrm{~K}$ ), and for $\sigma_{l} \sim 0.5 \sigma_{b}$ is expected to be appreciable. During the VIRGO antenna life-time (a few years) the suspension wire length $l$ is likely to increase linearly with time, $\delta l / l=\mu t$ (the rate $\mu$ being of the Arrhenius type). Every glitch of the suspended 
mirrors determines a variation of the cavity length. In fact, keeping the cavity mirrors parallel at distance $L=3 \mathrm{Km}$ requires tilting each mirror by an angle $\theta=310^{-4} \mathrm{rad}$ with respect to the vertical. Our antenna is sensitive to individual glitches as small as $10^{-14} \mathrm{~m}$ (oscillation bursts with frequency of $6.5 \mathrm{~Hz}$ and quality factor of $310^{3}$ ), that is much smaller than the creep step estimate $q_{s}=10^{-12} \mathrm{~m}$ for the steel samples tested so far. Moreover, if we assume that the creep process is due to a random superposition of independent glitches $q_{s}$ with Poisson rate constant $\lambda$, we easily come up with a new source of mechanical shot noise $\Delta L(t)$ corresponding to a background GW signal

$$
\tilde{h}(\nu)=\theta(6.5 \mathrm{~Hz} / \nu)^{2}\left(q_{s} / L\right) \sqrt{\lambda} / 2 \pi \nu
$$

where $\nu \gg 6.5 \mathrm{~Hz}, L=3 \mathrm{Km}$ and $\lambda$ is to be determined as a function of $\sigma_{l}$ and $T$. On inserting our preliminary result $\mu \simeq 10^{-11} \mathrm{~s}^{-1}$ and our estimate for $q_{s}$, we conclude that $\tilde{h}(\nu) \simeq$ $2.510^{-20}(6.5 \mathrm{~Hz} / \nu)^{3}$ : the mechanical shot noise (not plotted in Fig. 1) is large compared to the thermal (dislocation) noise in loaded wires. Morover, signals at $6.5 \mathrm{~Hz}$ must be discarded in the search for GW events at low frequencies: the analysis of the creep related bursts will provide us with an extremely accurate nanometric statistics of the creep mechanism.

(3) clamping technique. The VIRGO design requires mirror suspensions with quality factor $Q \geq$ $10^{6}$. For the chosen pendulum geometry (a cylinder hung by two wire loops) and the low-loss materials available to-date, say $\phi \simeq 310^{-4}$, we expect a best $Q$ of $510^{6}$, whereas the highest $Q$ measured in laboratory tests, so far, is $510^{5}$. The $Q$ defect may be due to: (i) an undetected load dependence of the wire loss angle; (ii) poor clamp performance [7] and/or wire-mirror friction; (iii) spurious effects in our experimental apparatus (or, possibly, a combination of the three). A better understanding of the dissipation processes in the vicinity to the clamps is required: the stick-andslip friction of the wires in the clamps can be viewed as an additional source of $1 / f$ noise, which corresponds to an $\omega$ independent contribution to the total loss angle [6]. Moreover, due to the heavy load, the wire bending is limited to a small regions close to the clamps, thus making plastic deformation effects not totally negligible.

\section{References}

[1] Thorne K. S., in 300 Years of Gravitation (CUP, Cambridge, 1987), p. 330.

[2] VIRGO Collaboration, Final Design, Version 0. (1995); Giazotto A., Phys. Rep. 182 (1989) 365.

[3] Saulson P. R., Phys. Rev. D42 (1990) 2437.

[4] Quinn T. J., Speake C. C., Tew W., Davis R. S. and Brown L. M., Phys. Lett. A197 (1995) 197.

[5] Cagnoli G., Gammaitoni L., Marchesoni F. and Segoloni D., Philos. Mag. 68 (1993) 865.

[6] Marchesoni F. and Patriarca M., Phys. Rev. Lett. 72 (1994) 4101.

[7] Cagnoli G., Gammaitoni L., Kovalik J., Marchesoni F. and Punturo M., Phys. Lett. A213 (1996) 245. 